\title{
ACRL Midwinter meetings in New Orleans
}

\author{
A tentative schedule
}

$\mathrm{E}$ d. note: ALA's Midwinter Meeting takes place from January 18-23, 2002, in New Orleans. This list of meeting times was current as we went to press. Be sure to check the program book for final times and locations of meetings.

\section{ACRL Board of Directors}

Board Update: Friday, Jan. 18, 9:00-11:30 a.m.

Leadership Council: Friday, Jan. 18, 2:004:00 p.m.

First meeting: Sunday, Jan. 20, 2:00-4:00 p.m.

Joint meeting with Budget \& Finance Committee: Monday, Jan. 21, 8:00-9:00 a.m.

Second meeting: Tuesday, Jan. 22, 1:004:30 p.m.

\section{ACRL General}

Digital Reference: Trends, Techniques, and Changes: Friday, Jan. 18, 1:00-5:00 p.m. (pre-registration required)

Experience the Power of Collaboration workshop: Friday, Jan. 18, 8:00 a.m.-5:30 p.m. (pre-registration required)

Focus on the Future Task Force: Monday, Jan. 21, 8:30-11:00 a.m. (pre-registration required)

IMIS Grant Participants Meeting: Thursday, Jan. 17, 8:30 a.m. $-4: 00$ p.m.

Leadership Council: Friday, Jan. 18, 2:004:00 p.m.

Presidential Candidate's Forum: Sunday, Jan. 20, 11:30-1:30 p.m.

President's Program Discussion Group and Meeting: Monday, Jan. 21, 9:30 a.m.-12:30 p.m. Topic: Learning Communuities. (see sidebar page 1082)

Scholarly Publishing and Academic Resources Coalition (SPARC)/ACRL Forum: Saturday, Jan. 19, 9:30-11:00 a.m.

\section{ACRL Divisional Committees}

Academic/Research Librarian of the Year Award Selection: Sunday, Jan. 20, 9:3012:30 p.m. (closed)

Appointments: Friday, Jan. 19, 2:00-5:30 p.m.; Monday, Jan. 21, 2:00-4:00 p.m.; Saturday, (closed)

Best Practices Project Team: Monday, Jan. 21, 9:00 a.m.-3:30 p.m.

Budget and Finance: Saturday, Jan. 19, 8:30 a.m.-12:30 p.m.; Sunday, Jan. 20, 8:0011:00 a.m.; Monday, Jan. 21, 9:30-11:30 a.m.

Bylaws Committee: Saturday, Jan. 19, 9:30-11:00 a.m.; Monday, Jan. 21, 9:30-11:00 a.m.

Conference Program Planning-Atlanta, 2002: Saturday, Jan. 19, 8:00-9:00 a.m.

Conference Program PlanningToronto, 2003: Saturday, Jan. 19, 4:30-5:30 p.m.

Copyright: Sunday, Jan. 20, 9:30-12:30 p.m.

Council of Liaisons: Saturday, Jan. 19, 11:30 a.m.-1:00 p.m.

Doctoral Dissertation: Saturday, Jan. 19 , 9:30 a.m. $-12: 30$ p.m. (closed)

Effective Practices: Saturday, Jan. 19, 2:00-5:30 p.m.;

Ethics: Sunday, Jan. 20, 4:30-6:00 p.m.

Government Relations: Monday, Jan. 21, 9:30 a.m.-12:30 p.m. 
Information Literacy Advisory: Saturday, Jan. 19, 4:30-5:30 p.m.

Information Literacy Joint committee with AASL: Saturday, Jan. 19, 4:00-6:00 p.m.

Institute for Information Literacy Advisory: Friday, Jan. 18, 2:00-5:00 p.m.; Sunday, Jan. 20, 8:30 a.m.-12:30 p.m.

Institute for Information Literacy Best Practices: Monday, Jan. 21, 9:00 a.m.-3:30 p.m.

Intellectual Freedom: Saturday, Jan. 19, 11:30 a.m. $-12: 30$ p.m.

International Relations: Sunday, Jan. 20, 8:30-11:00 a.m.

Lazerow Fellowship Committee: Saturday, Jan. 19, 2:00-5:30 p.m. (closed)

Membership: Saturday, Jan. 19, 9:00-4:00 p.m.
National Conference Executive Committee-Charlotte, 2003: Saturday, Jan. 19, 11:30 a.m.-1:30 p.m.

National Conference Subcommittees Charlotte, 2003: Monday, Jan. 21, 8:30 a.m.12:30 p.m.

Nominations, 2003: Monday, Jan. 21 8:00-9:00 a.m.

President's Program Planning -Atlanta, 2002: Saturday, Jan. $19,9: 00$ a.m. $-5: 30$ p.m; Monday, Jan. 21, 9:30 a.m.-12:30 p.m.

President's Program PlanningToronto, 2003: Saturday, Jan. 19, 2:00-4:00 p.m.; Monday, Jan. 21, 8:30-11:00 a.m.

Professional Development: Saturday, Jan 19, 9:30 a.m.-12:30 p.m.

Publications: Saturday, Jan. 19, 8:00-9:00 a.m.; Monday, Jan. 21, 8:30-11:00 a.m.

\section{ACRL Discussion Groups}

$E d$. note: Section discussion groups are listed with their sections.

Australian, Canadian, and New Zealand Studies: Sunday, Jan. 20, 11:30 a.m.-12:30 p.m. Topic: Quebec as a Conduit to Europe for North American Library Practices.

Consumer and Family Studies: Sunday, Jan. 20, 4:30-5:30 p.m.

Criminal Justice/Criminology: Saturday, Jan. 19, 4:30-5:30 p.m.

Electronic Reserves: Sunday, Jan. 20 , 8:30-11:00 a.m.

Electronic Text: Saturday, Jan. 19, 2:004:00 p.m. Topic: Electronic Texts in the 21st Century.

Fee-based Information Service Centers in Academic Libraries (FISCAL): Saturday, Jan. 19, 2:00-4:00 p.m. Topic: Making the Most of Your Resources-Using ILL Management Software in a Fee-Based Setting.

Heads of Public/Readers Services: Sunday, Jan, 20, 4:30-5:30 p.m. Topic: Partnerships-Yesterday, Today, and Tomorrow.

Library Development: Saturday, Jan. 19, 8:30-11:00 a.m. Topic: Fundraising and Detelopment Programs for the University and College Libravies.

Media Resources: Sunday, Jan. 20, 9:3011:00 a.m.

Medium-Sized Academic Libraries: Sunday, Jan. 20, 9:30-11:00 a.m. Topic: Dis- tance Education, Electronic Reference, and Library Service.

MLA International Bibliography in Academic Libraries: Saturday, Jan. 19, 9:30-11:30 a.m.

Personnel Administrators and Staff Development : Saturday, Jan. 19, 9:3011:00 a.m.; Sunday, Jan. 20, 9:30-11:00 a.m. Topic: Human Resources in Academic Libraries: Recruitment and Retention.

Philosophical, Religious, and Theological Studies Discussion Group: Sunday, Jan. 20, 4:30-6:30 p.m. Open Discussion.

Popular Culture and Libraries: Sunday, Jan. 20, 4:40-5:30 p.m.

President's Program Discussion Group: Monday, Jan. 21, 9:30 a.m.-12:30 p.m. Topic: Learning Communities.

Research Discussion Group: Saturday, Jan. 19, 2:00-5:30 p.m.

Scholarly Publishing and Academic Resources Coalition (SPARC)/ACRL Forum: Saturday, Jan. 19, 9:30-11:00 a.m. Topic:

Sports and Recreation Discussion Group: Saturday, Jan. 19, 8:00-9:00 a.m.

Team-Based Organizations: Sunday, Jan. 20, 11:30 a.m.-12:30 p.m.

Undergraduate Libraries: Monday, Jan. 21, 8:30-11:00 a.m. 
Racial and Ethnic Diversity: Saturday, Jan. 19, 9:30-11:00 a.m.

Research: Saturday, Jan. 19, 11:30 a.m.12:30 p.m.

Scholarly Communications Task Force: Sunday, Jan. 20, 8:00-11:00 a.m.

Section Newsletter Editors: Sunday, Jan. 20, 2:00-4:00 p.m.

Standards and Accreditation: Saturday, Jan. 19, 2:00-5:30 p.m.

Statistics: Friday, Jan. 18, 4:30-5:15 p.m.

Status of Academic Librarians: Saturday, Jan. 19, 2:00-5:30 p.m.

\section{ACRL Chapters Council}

ACRL Presidential Candidates Forum: Sunday, Jan. 20, 11:30 a.m.-1:00 p.m.

Business and Orientation: Sunday, Jan. 20, 8:30-11:00 a.m.

\section{ACRL Editorial Boards}

CHOICE: Sunday, Jan. 20, 8:30-11:00 a.m. CHOICE Reviews Online focus group: Monday, Jan. 21, 7:00-8:30 a.m.

College \& Research Libraries: Saturday, Jan. 19, 11:30 a.m.-12:30 p.m.

College \& Research Libraries News: Sunday, Jan. 20, 8:30-11:00 a.m.

New Publications Advisory Board: Sunday, Jan. 20, 11:30 a.m.-12:30 p.m.

Publications in Librarianship: Saturday, Jan. 19, 2:00-4:00 p.m.

RBM: Sunday, Jan. 20, 9:30 a.m.-12:30 p.m.

\section{Meet Cannon and Popko-the candidates for ACRL President}

Hear Tyrone Cannon and John Popko, the official candidates for ACRL vice-president/president-elect, speak at the ACRL Presidential Candidates Forum on Sunday, January 20, 2001, 11:30 a.m.-1:00 p.m., during the Midwinter Meeting in New Orleans, Louisiana.

Cannon is the library dean at the University of San Francisco. Popko is the university librarian at Seattle University.

The forum, organized by the ACRL Chapters Council, will be followed by a light lunch, sponsored by EBSCO Subscription Services.

\section{ACRL Sections}

Sections Council: Friday, Jan. 18, 4:30-5:30 p.m.

\section{African-American Studies Librarians Section}

Executive: Saturday, Jan. 19, 9:30-11:00 a.m.

Standing Committees: Saturday, Jan. 19, 8:00-9:00 a.m

Membership: Saturday, Jan. 19, 2:00-4:00 p.m.

Cataloging Issues Discussion Group: Sunday, Jan. 20, 9:30 a.m.-11:00 a.m.

\section{Asian, African and Middle Eastern Section}

Executive: Saturday, Jan. 19, 9:30 a.m.-12:30 p.m.

Program Planning, 2002: Friday, Jan. 18, 9:30-11:30 a.m.

Publishers Liaison Committee: Friday, Jan. 18, 2:00-4:00 p.m.

\section{Anthropology and Sociology Section}

Executive: Saturday, Jan. 19, 8:00-9:00 a.m.; Monday, Jan. 21, 9:30 a.m.-12:30 p.m.

Anthropology Librarians Discussion Group: Sunday, Jan. 20, 4:30-5:30 p.m.

Bibliography: Saturday, Jan. 19, 9:30-11:00 a.m.; Sunday, Jan. 20, 9:30-11:00 a.m.

Conference Program Planning-Toronto, 2003: Monday, Jan. 21, 8:00-9:00 a.m

Conference Program Planning-Atlanta, 2002: Sunday, Jan. 20, 8:00-9:00 a.m.

Liaison: Saturday, Jan. 19, 9:30-11:00 a.m Membership: Sunday, Jan. 20, 2:00-4:00 p.m.

Nominating: Saturday, Jan. 19, 9:30-11:00 a.m. (closed)

Publications: Saturday, Jan. 19, 2:00-4:00 p.m.

Review and Planning: Saturday, Jan. 19, 9:30-11:00 a.m.

Sociology Librarians Discussion Group: Saturday, Jan. 19, 11:30 a.m.-12:30 p.m.

Subject and Bibliographic Access: Saturday, Jan. 19, 9:30-11:00 a.m.

\section{Arts Section}

Executive and Membership: Sunday, Jan. 20, 9:30-11:00 a.m.

All Committees: Saturday, Jan. 19, 9:30 a.m12:30 p.m.

Dance Librarians Discussion Group: Sunday, Jan. 20, 2:00-4:00 p.m. 
Film/Broadcast Studies Librarians Discussion Group: Sunday, Jan. 20, 2:00-4:00 p.m.

Performing Arts Discussion Group: Sunday, Jan. 19, 8:00-9:00 a.m.

\section{Community and Junior College Libraries Section}

Executive: Saturday, Jan. 19, 11:30 a.m.12:30 p.m.; Monday, Jan. 21, 8:30-11:00 a.m.

All Committees: Saturday, Jan. 19, 8:30-11:00 a.m.

NCLR: Sunday, Jan. 20, 2:00-4:00 p.m.

Standards: Friday, Jan. 18, 2:00-4:00 p.m.

\section{College Libraries Section}

Executive: Saturday, Jan. 19, 9:30-11:00 a.m.; Monday, Jan. 21, 2:00-4:00 p.m.

CLIP Notes: Saturday, Jan. 19, 11:30 a.m12:30 p.m.

College Librarians and Staff Discussion Group: Sunday, Jan. 20, 4:30-5:30 p.m.

College Library Directors' Discussion Group: Sunday, Jan. 20, 2:00-4:00 p.m.

Committee on the status of College Librarians (Ad Hoc ): Saturday, Jan. 19, 9:30 a.m12:30 p.m.

Communications: Saturday, Jan. 19, 2:004:00 p.m.

Conference Program Planning-Atlanta, 2002: Sunday, Jan. 20, 8:30-11:00 a.m.

Conference Program Planning-Toronto, 2003: Saturday, Jan. 19, 2:00-4:00 p.m.

Continuing Education: Monday, Jan. 21, 9:30-11:00 a.m.

Leadership: Monday, Jan. 21, 9:30-11:00 a.m. Medium-Sized Academic Libraries Discussion Group: Sunday, Jan. 20, 9:30-11:00 a.m.

Membership: Monday, Jan. 21, 8:00-9:00 a.m.

Research for College Librarianship: Sunday, Jan. 20, 9:30 a.m.-12:30 p.m.

Standards: Saturday, Jan. 19, 11:30 a.m12:30 p.m.; Sunday, Jan. 20, 4:30-5:30 p.m.

\section{Distance Learning Section}

Executive: Sunday, Jan. 20, 11:30 a.m.-12:30 p.m.; Monday, Jan. 21, 11:30 a.m.-12:30 p.m.

All Committees: Sunday, Jan. 20, 8:00-11:00 a.m.

Discussion Group: Monday, Jan. 21, 8:3011:00 a.m.

\section{Education and Behavioral Sciences Section}

Executive: Friday, Jan. 18, 8:00-10:00 p.m.
Advisory Council: Sunday, Jan. 20, 8:3011:00 a.m.

Conference Program Planning-Atlanta, 2002: Sunday, Jan. 20, 4:30-6:30 p.m.

Conference Program Planning-Toronto, 2003: Sunday, Jan. 20, 4:30-5:30 p.m.

Consolidated Committee Meeting (Curriculum Materials, Distinguished Librarian Award, Instruction for Educators, Membership and Orientation, and Reference Sources and Services): Saturday, Jan. 19, 9:30 a.m.-12:30 p.m.

Current Topics Discussion Group: Saturday, Jan. 19, 2:00-4:00 p.m.

Curriculum Materials Centers Standards/ Guidelines Committee (Ad Hoc): Saturday, Jan. 19, 8:00-11:00 a.m.

Digital Publication of the CMC Management Guide (Ad Hoc): Saturday, Jan. 19, 8:00-9:30 a.m.

Historical Textbook and Curriculum Collections Directory (Ad Hoc): Sunday, Jan. 20, 2:004:00 p.m.

Psychology/Psychiatry: Saturday, Jan. 19, 8:30-11:00 a.m.

Publications \& Communications: Saturday, Jan. 19, 8:00 a.m.-12:30 p.m.

Social Work/Social Welfare: Saturday, Jan. 19, 4:30-6:30 p.m.

\section{Instruction Section}

Section Dinner: Friday, Jan. 18, 6:00-9:00 p.m. Ralph and Kaccoo's, \$30. Contact Marji McKenzie at MMackenz@grcc.ctc.edu to resene a. space.

Executive: Saturday, Jan. 19, 8:00-9:00 a.m.; Tuesday, Jan. 22, 8:30-11:00 a.m.

Advisory Council: Saturday, Jan. 19, 9:3011:00 a.m.; Saturday, Jan. 19, 11:30 a.m.-12:30 p.m.; Monday, Jan. 21, 2:00-4:00 p.m.

Awards: Saturday, Jan. 19, 2:00-4:00 p.m.; Sunday, Jan. 20, 9:30-11:00 a.m. (closed)

Communication: Saturday, Jan. 19, 2:00-4:00 p.m.; Sunday, Jan. 20, 9:30-11:00 a.m.

Conference Program Planning-Atlanta, 2002: Saturday, Jan. 19, 2:00-4:00 p.m.; Monday, Jan. 21, 9:30-11:00 a.m.

Conference Program Planning-Toronto, 2003: Sunday, Jan. 20, 9:30-11:00 a.m.; Monday, Jan. 21, 9:30-11:00 a.m.

Education: Saturday, Jan. 19, 2:00-4:00 p.m.; Sunday, Jan. 20, 9:30-11:00 a.m.

Emerging Technologies in Instruction: Saturday, Jan. 19, 2:00-4:00 p.m.; Sunday, Jan. 20, 9:30-11:00 a.m. 
Immersion Alumni: To be announced

Instruction for Diverse Populations: Saturday, Jan. 19, 1:00-4:00 p.m.; Sunday, Jan. 20, 4:30-5;30 p.m.

Management for Instruction Services: Saturday, Jan. 19, 2:00—4:00 p.m.; Sunday, Jan. 20, 9:30-11:00 a.m.

Membership: Saturday, Jan. 19, 2:00-4:00 p.m.

Nominating, 2003: Saturday, Jan. 19, 2:004:00 p.m.; Monday, Jan. 21, 9:30-11:00 a.m. (closed)

Planning: Saturday, Jan. 19, 2:00-4:00 p.m.; Monday, Jan. 21, 9:30-11:00 a.m.

Policy: Saturday, Jan. 19, 2:00-4:00 p.m.; Monday, Jan. 21, 9:30-11;00 a.m.

Preconference Planning-Atlanta, 2002: Saturday, Jan. 19, 2:00-4:00 p.m.; Monday, Jan 21, 9:30-11:00 a.m.

Preconference Planning-Toronto, 2003 Saturday, Jan. 19, 2:00-4:00 p.m.; Monday, Jan 21, 9:30-11:00 a.m.

Research and Scholarship: Saturday, Jan. 19 , 2:00-4:00 p.m.; Monday, Jan. 21, 9:30-11:00 a.m.
Teaching Methods: Saturday, Jan. 19, 2:004:00 p.m.; Sunday, Jan. 20, 2:00-4:00 p.m.

\section{Law and Political Science Section}

All Committees/General Membership Meeting: Saturday, Jan. 19, 2:00-5:30 p.m.

Library Instruction Discussion Group: Sunday, Jan. 20, 2:00-4:00 P.m.

Marta Lange/CQ Award: Sunday, Jan. 20, 4:30-5:30 p.m. (closed)

\section{Literatures in English Section}

Executive: Saturday, Jan. 19, 2:00-4:00 p.m.; Monday, Jan. 21, 2:00-4:00 p.m.

General Membership Meeting: Sunday, Jan. 20, 2:00-4:00 p.m.

All Committees: Monday, Jan. 21, 9:30-11:00 a.m.

Nineteenth Century Discussion Group: Sunday, Jan. 20, 4:30-5:30 p.m.

Nominating: Sunday, Jan. 20, 9:30-11:00 a.m.

Reference Discussion Group: Sunday, Jan. 20, 9:30-11:00 a.m.

\section{Learning communities and ACRL}

Everyone is invited to join an informal discussion forum during ALA's Midwinter Meeting that will focus on ACRL as a learning community. ACRL President Mary Reichel will lead the discussion and highlight her theme of learning communities and excellence in academic libraries.

In the November 2001 issue of CGRL News, Don Frank, Sarah Beasley, and Susan Kroll provided an overview of learning communities in higher education with examples of successful programs that involved librarians. ${ }^{1}$ Learning communities can be characterized as the coming together of individuals in the spirit of exploration and learning in an interdisciplinary environment. Along with the deep learning that occurs over time is a development of a mutual responsibility for the community.'

The Monday forum at Midwinter will focus on how ACRL, acting as a learning community through its sponsored programs and activities, has promoted excellence in academic library services. Specific examples of how libraries have benefited by ACRL programs will be presented. This is a great op- portunity for ACRL members to come together as a learning community to share their stories about the kinds of programs ACRL has supported and to discuss their participation in specific learning communities on their campuses. Come and find out more about learning communities and $\Lambda$ CRL. Network with other academic librarians. The discussion forum will be held on Monday, January 21, 2002, from 9:30-11 a.m. Check the Midwinter Meeting calendar for location.-Jill Newby, Science Librarian. Weber State University, jnewby@weberedu.

\section{Notes}

1. Donald G. Frank, Sarah Beasley, and Susan Kroll. "Learning Communities: Opportunities for Collaborative Excellence," College and Research Libraries Neus 62, no. 10 (November 2001).

2. Faith Gabelnick, Jean MacGregor, Roberta S. Matthews, and Barbara Leigh Smith, Learning Communities: Greating Connections Among Students, Faculty, and Disciplines (New Directions for Teaching and Leaning, no. 41, San Francisco: Jossey Bass, 1990): 60. 


\section{Rare Books and Manuscrípts Section}

Executive: Monday, Jan. 21, 8:30-11:00 a.m.

Bibliographic Standards: Saturday, Jan. 19, 8:30 a.m.-12:30 p.m.; Sunday, Jan. 20, 8:3011:00 a.m.

Bibliographic Standards/Thesaurus Subcommittee: Friday, Jan. 18, 2:00-5:30 p.m.

Budget and Development: Saturday, Jan. 19, 2:00-4:00 p.m.

Committee to Revise Standards for Ethical Conduct: Friday, Jan. 18, 8:00-10:00 p.m.

Conference Development: Sunday, Jan. 20, 8:30-11:00 a.m.

Conference Program Planning-Atlanta, 2002: Saturday, Jan. 19, 11:30 a.m.-12:30 p.m.

Conference Program Planning-Toronto, 2003: Sunday, Jan. 20, 9:30-11:00 a.m.

Curators and Conservators Discussion Group: Sunday, Jan. 20, 8:30-11:00 a.m.

Exhibition Catalog Awards: Saturday, Jan. 19, 8:30 a.m.-12:30 p.m. (closed); Sunday, Jan. 20, 8:30-11:00 a.m

Hearing on Guidelines Regarding Thefts in Libraries: Saturday, Jan. 19, 8:00-10:00 p.m.

Information Exchange: Sunday, Jan. 20, 4:30-5:30 p.m.

MARC for Special Collections Discussion Group: Sunday, Jan. 20, 11:30 a.m.-12:30 p.m.

Manuscripts and Other Formats Discussion Group: Sunday, Jan. 20, 2:00-4:00 p.m.

Membership and Professional Development: Saturday, Jan. 19, 8:30-11:00 a.m.

Nominating: Saturday, Jan. 19, 11:30 a.m12:30 p.m. (closed)

Preconference Program Planning-Atlanta, 2002: Saturday, Jan. 19, 8:30-11:00 a.m.

Preconference Program Planning-Toronto, 2003: Saturday, Jan. 19, 11:30 a.m.-12:30 p.m.

Public Services Discussion Group: Sunday, Jan. 20, 2:00-4:00 p.m.

Publications: Saturday, Jan. 19, 2:00-4:00 p.m.

Security: Sunday, Jan. 20, 2:00-4:00 p.m.

Seminars: Saturday, Jan. 19, 2:00-4:00 p.m.

\section{Slavic and East European Section}

Block Committee Meeting: Automated

Bibliographic Control, Conference Program Planning-2002, Continuing Education, Electronic Resources, Newsletter, and Preservation: Saturday, Jan. 19, 9:00 a.m.-5:30 p.m.

\section{Science and Technology Section}

Council: Friday, Jan. 18, 8:00-10:00 p.m.;
Monday, Jan. 21, 8:30-11:00 a.m.

Comparison of Science and Technology Libraries: Saturday, Jan. 19, 8:30-11:00 a.m.

Conference Program Planning-Atlanta, 2002: Saturday, Jan. 19, 8:30 a.m.-12:30 p.m.

Conference Program Planning, Toronto2003: Saturday, Jan. 19, 9:30-11:00a.m.

Continuing Education: Sunday, Jan. 20, 9:3011:00 a.m.

Discussion Group Chairs: Saturclay, Jan. 19, 11:30 a.m.-12:30 p.m.

General Discussion Group: Saturday, Jan. 19, 2:00-4:00 p.m.

Government Information: Sunday, Jan. 20, 8:00-11:00 a.m.

Heads of Science and Technology Libraries Discussion Group: Sunday, Jan. 20, 8:00-10:00 p.m.

Membership and Recruitment: Sunday, Jan. 20, 8:00-9:00 a.m.

Oberly Award: Saturday, Jan. 19, 8:00 a,m12:30 p.m. (closed)

Organization and Planning: Saturday, Jan. 19, 2:00-4:00 p.m.

Publications: Saturday, Jan. 19, 9:30-11:00 a.m.

Publisher/Vendor Relations Discussion Groups: Sunday, Jan. 20, 9:30-11:00 a.m.

Research Committee: Saturday, Jan. 19, 2:004:00 p.m.

Science Databases Discussion Group: Sunday, Jan. 20, 9:30-11:00 a.m.

Subject and Bibliographic Access: Saturday, Jan. 19, 9:30 a.m.-11:00 a.m.

\section{University Libraries Section}

Executive: Saturday, Jan. 19, 8:30-11:00 a.m.; Monday, Jan. 21, 8:30-11:00 a.m.

Campus Administration and Leadership Discussion Group: Saturday, Jan. 19, 11:30 a.m12:30 p.m.

Communications: Saturday, Jan. 19, 11:30 a.m.-12:30 p.m.

Conference Program Planning, 2002: Saturday, Jan. 19, 11:30 a.m.-12:30 p.m.; Monday, Jan. 21, 11:30 a.m.-12:30 p.m.

Conference Program Planning, 2003: Sunday, Jan. 20, 9:30 a.m.-12:30 p.m.

Current Topics Discussion Group: Saturday, Jan. 19, 2:00-4:00 p.m.

Organization and Bylaws: Sunday, Jan. 20, 9:30-11:00 a.m.

Policy and Planning: Sunday, Jan. 20, 9:00 a.m. $-5: 30$ p.m. 
Public Service Directors of Large Research Libraries Discussion Group: Sunday, Jan. 19, 2:00-4:00 p.m.

Standards/Guidelines Review Committee: Saturday, Jan. 19, 11:30 a.m.-12:30 p.m.; Sunday, Jan. 20, 2:00-4:00 p.m.; Monday, Jan. 21, 2:00-4:00 p.m.

Urban 13 Libraries Group: Saturday, Jan. 19, 4:30-5:30 p.m.

\section{Western European Studies Section}

Executive/General Membership: Tuesday, Jan. 22, 8:30 a.m.-12:30 p.m.

Cataloging Discussion Group: Monday, Jan. 21, 11:30 a.m. $-12: 30$ p.m.

Classics, Medieval, and Renaissance Discussion Group: Saturday, Jan. 19, 11:30 a.m.-12:30 p.m.

College and Medium-Sized Libraries Discussion Group: Sunday, Jan. 20, 4:30-5:30 p.m.

Conference Program Planning-Atlanta, 2002: Sunday, Jan. 20, 11:30 a.m.-12:30 p.m.

Conference Program Planning-Toronto, 2003: Monday, Jan. 21, 11:30 a.m.-12:30 p.m.

Germanists Discussion Group: Sunday, Jan. 20, 9:30-11:00 a.m.

International Conference Planuing (Paris)-
2004: Sunday, Jan. 20, 8:00-10:00 p.m.

Membership: Saturday, Jan. 19, 9:30-11:00 a.m.

Nominations: Saturday, Jan. 19, 8:00-9:00 a.m.

Publications: Monday, Jan. 21, 2:00-4:00 p.m.

Research and Planning: Saturday, Jan. 19, 2:00-4:00 p.m.

Romance Languages Discussion Group: Saturday, Jan. 19, 11:30 a.m.-12:30 p.m.

Scandinavian Discussion Group: Saturday, Jan. 19, 11:30 a.m.-12:30 p.m.

Social Sciences \& History Discussion Group: Monday, Jan. 21, 9:30-11:00 a.m.

\section{Women's Studies Section}

Executive: Monday, Jan. 21, 11:30 a.m.-1:30 p.m.

All Committees: Sunday, Jan. 20, 9:30-11:00 a.m.

Awards: Friday, Jan. 18, 4:30-5:30 p.m.; Sunday, Jan. 20, 9:30 a.m.-11:00 a.m.

Discussion Meeting: Monday, Jan. 21, 9:3011:00 a.m.

General Membership: Sunday, Jan. 20, 4:305:30 p.m.

\section{Elegant Solutions for Preservation}

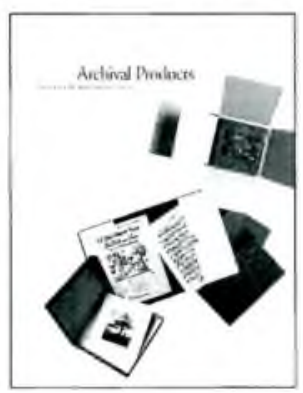

Call

for a complete catalog

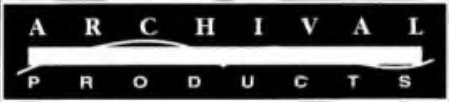

A Division Or LIBAAMV BINDING SEKVICE

\begin{tabular}{ll} 
Pamphlet Binders & Bound Four Flap Enclosures \\
\hline Music Binders & Archival Binders \\
\hline Archival Folders & Archival Boards \\
\hline Manuscript Folders & Adhesives \\
\hline Hinge Board Covers & Bookkeeper \\
\hline Academy Folders & Century Boxes \\
\hline Newspaper/Map Folders & Record Album Enclosures \\
\hline Polypropylene Sheet \& Photo Protectors & Conservation Cloths \\
\hline
\end{tabular}

Conservation \& Preservation Tapes

\section{ARCHIVAL PRODUCTS \\ A Division of Library Binding Service}

P.O. Box 1413. Des Moines, lowa 50305-1413

800.526.5640 Fax 888.220.2397

custserv(harchival.com htp://www.archival.com 


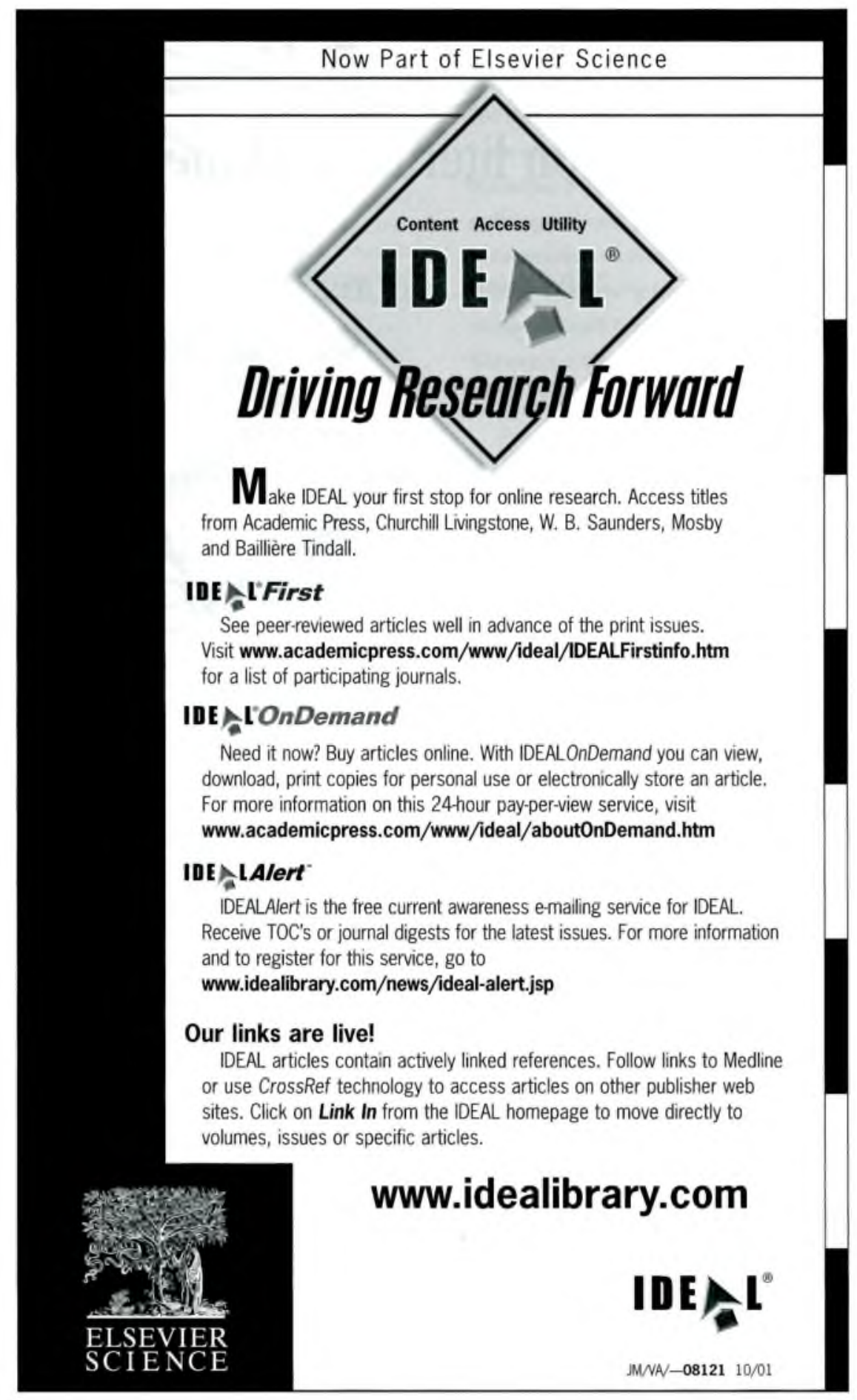

